\title{
Effect of Corn Peptides in Combination with Exercise on Weight Reduction, Plasma Lipid, Chemerin and Lp- $\mathrm{PLA}_{2}$ in Overweight and Obese Females
}

\author{
Yin $\mathrm{Wu}^{1}$, Liang Yin ${ }^{2}$, Xiaohui Wang ${ }^{2, *}$, Shujie Lou ${ }^{2}$ \\ ${ }^{1}$ Department of Computer and Statistics, Shanghai University of Sport, Shanghai, China \\ ${ }^{2}$ Department of Sport Science, Shanghai University of Sport, Shanghai, China \\ *Corresponding author: wangpan96@126.com
}

Received June 20, 2014; Revised August 10, 2014; Accepted August 13, 2014

\begin{abstract}
Our previous work has demonstrated better weight reduction and plasma lipid improvement roles of corn peptides (CP) in combination with aerobic exercise than aerobic exercise in obese rats, the purpose of this paper is to explore if the better effectiveness of CP combined with exercise also exist in overweight and slight obese young females and to determine the possible role of adipokine chemerin and inflammation factor lipoprotein-associated phspholipase $A_{2}$ (Lp-PLA $A_{2}$. Twenty-six young female (18-21 years old, body fat percentage: $29.15 \% \pm 2.71 \%$ and body mass index (BMI): 23.92 \pm 1.78 ) were allocated randomly to four groups: exercise 1 group (E1, n=6), CP+E1 group ( $(n=7)$, E2 group ( $n=6)$ and $C P+E 2$ group $(n=7)$. The females in $E 1$ and $C P+E 1$ groups did 1 hour aerobics 3 times a week, while the same aerobics were done but 5 times a week by E2 and $\mathrm{CP}+\mathrm{E} 2$ groups. The exercise lasts for four weeks and $10 \mathrm{~g}$ of CP were taken orally by the females in the two CP groups every night after meal. Similar power placebo was administered to the non-CP treated females. The diet of these females kept unchanged during the 4 weeks. The body weight, BMI, body fat percentage and plasma lipid parameters including triglyceride (TG), total cholesterol (TC), high density lipoprotein-cholesterol (HDL-C), low density lipoprotein-cholesterol (LDL-C), apolipoprotein a (Apo-A), apolipoprotein b (Apo-B) and the ratios of LDL-C/HDL-C and Apo-B/Apo-A as well as plasma levels of chemerin and Lp-PLA $A_{2}$ were determined pre and post 4 weeks. Compared to E1 group, all the above parameters did not change in $\mathrm{CP}+\mathrm{E} 1$ group after 4 weeks. Except for an obvious reduction of body fat percentage (from $29.44 \% \pm 2.65 \%$ to $27.93 \% \pm 2.56 \%$ vs from $27.95 \% \pm 3.38 \%$ to $27.47 \% \pm 3.88 \%$, $p<0.05$ ) were found in $\mathrm{CP}+\mathrm{E} 2$ group, other changes were still not observed compare with that of E2. It's worth noting that obvious reduction of chemerin was found in individuals with abnormal lipid parameters return to normal and no change of chemerin was showed in a female whose abnormal lipid index kept stable after treatment with CP combined with exercise. In conclusion, only when frequent aerobic exercise was performed did CP in combination with aerobic exercise promote significantly the decrease of body fat percentage in overweight and slight obese females. The fat loss resulted from CP in combination with exercise is not related to the plasma levels of chemerin and Lp-PLA, which might be attributed to no change of blood lipid parameters within normal ranges in overweight and slight obese females. Although no difference of chemerin between groups, changes of plasma chemerin in individual females with abnormal lipid parameters suggested that $\mathrm{CP}$ in combination with exercise might reverse the abnormal plasma levels of TG, TC and LDL-C by decreasing the plasma levels of chemerin.
\end{abstract}

Keywords: $C P$, chemerin, body fat percentage, $L p-P L A_{2}$

Cite This Article: Yin Wu, Liang Yin, Xiaohui Wang, and Shujie Lou, "Effect of Corn Peptides in Combination with Exercise on Weight Reduction, Plasma Lipid, Chemerin and Lp-PLA 2 in Overweight and Obese Females." Journal of Food and Nutrition Research, vol. 2, no. 8 (2014): 485-490. doi: 10.12691/jfnr-2-8-9.

\section{Introduction}

Obesity is one of the main human epidemics today, leading to several chronic obesity-associated diseases such as hyperlipidemia, coronary atherosclerosis, diabetes and hypertension. The cause of obesity is very complicated results from interactions among a variety of factors, in which over-nutrition and insufficient energy consumption are the two main causes. Therefore diet control and aerobic exercise are emphasized to prevent and treat obesity, which are difficult to persist for obese persons because of tedious, time consuming and weak willpower. Therefore, specific nutrients and substances possessing weight reduction effects are becoming a focus of functional foods recently.

Corn is a major cereal crop throughout the world. Corn gluten meal containing approximately $60 \%$ protein is difficult to utilize in the food industry because of its low water solubility and amino acid imbalance [1]. Recently, corn gluten meal was hydrolyzed with a biochemical 
grade alcalase and the derived hydrolysates were purification to produce CP. Corn peptides (CP) is less than 2000 Da molecular weight and absorbed easily by digestive system. As bioactive peptides, CP attracts growing attention for its multiple functions such as angiotensin-converting enzyme inhibitor [2], alcohol metabolism enhancer [3] and antioxidant capacity [4]. Bong H.Y. reported the effect of CP on body weight reduction and improvement of lipid metabolism in obese rats [5], which makes $\mathrm{CP}$ an interesting area for further investigation. Our previous work has demonstrated that $\mathrm{CP}$ in combination with aerobic exercise could reduce the body weight, perirenal fat and plasma levels of triglyceride (TG), total cholesterol (TC) and LDL-C while no roles were found when only supplement of CP without exercise [6].

Chemerin is an adipose-derived signaling protein (adipokine) that regulates adipocyte differentiation and lipolysis as well as glucose homeostasis. Recent experimental and clinical data indicated that circulating chemerin expression and activation were elevated in numerous metabolic and inflammatory diseases including obesity $[7,8,9,10]$, metabolic syndrome [10,11,12], type 2 diabetes [12,13,14] and coronary artery disease [15]. Chemerin significantly decreased after bariatric surgery in obese patients undergoing bariatric surgery, accompanied by pronounced weight loss and improvements in parameters of lipid and glucose metabolism [16]. So chemerin may be a promising new target for the treatment of obesity, metabolic syndrome and type 2 diabetes [10,12]. Lipoprotein-associated phospholipase $A_{2}$ (Lp$\left.\mathrm{PLA}_{2}\right)$ is an inflammatory mediator involved in atherosclerosis [17]. Elevated levels of Lp-PLA 2 appear to be a strong risk factor for the severity of coronary atherosclerosis [18] and cardiovascular events in patients with coronary heart disease [19]. 4 months of low-calorie diet associated with weight loss in obese women resulted in the significant reduction of the plasma levels of Lp$\mathrm{PLA}_{2}$ [20].

Therefore the present study is to explore the effect of $\mathrm{CP}$ in combination with aerobic exercise on weight reduction, plasma lipid as well as plasma Lp-PLA $\mathrm{A}_{2}$ and chemerin in overweight and slight obese young females.

\section{Material and Methods}

\subsection{Subjects}

The criteria of overweight and obesity in adult females of Chinese were $24-27.9 \mathrm{~kg} / \mathrm{m}^{2}$ and $\geq 28 \mathrm{~kg} / \mathrm{m}^{2}$ by BMI or $25 \%-29.9 \%$ and $\geq 30 \%$ by body fat percentage, respectively. The body fat percentage of our twenty-six female subjects (18-21 years old) were $29.15 \% \pm 2.71 \%$ and BMI were $23.92 \pm 1.78 \mathrm{~kg} / \mathrm{m}^{2}$. The discrepancy of the two indicators was found in some female subjects, whose lower BMI might be result from less muscle mass. Considering the more concise in reflecting the fat mass or obesity, the body fat percentage criteria was selected in choosing subjects and our subjects were described as overweight or slight obese females. They were random divided into four groups: E1 group $(n=6), C P+E 1$ group $(n=7)$, E2 group $(n=6)$ and $C P+E 2$ group $(n=7)$.

\subsection{Analysis and Supplement of $\mathbf{C P}$}

CP was purchased from Ruibang biological technology co., ltd. (China). The molecular weight distribution and amino acid composition of CP were analyzed by test and analysis center of Jiangnan University (Jiangsu, China). $10 \mathrm{~g}$ of CP was taken orally by females in the two CP groups every night after meal. Similar power placebo was administered to the non-CP treated females.

\subsection{Exercise Protocol and Diet}

The females in $\mathrm{E} 1$ and $\mathrm{CP}+\mathrm{E} 1$ groups did aerobics for 1 hour, 3 times a week and last for 4 weeks, while the same aerobics were done but 5 times a week by E2 and CP+E2 groups. The intensity of this aerobics are moderate and the heart rates of these females were monitored and controlled at the range of $40 \%-60 \%$ heart rate reserve (about 125-150 beats/min) during doing aerobics, representing moderate aerobic exercise. The diet of these females kept unchanged during the 4 weeks.

\subsection{Determination of Body Weight, BMI and Body Fat Percentage}

The body weights and heights of these females at pre and post 4 weeks were detected by a scale and their BMI was calculated as the body weight divided by the squared height. Body fat percentages of these females were determined by body fat measuring instrument (Omron HBF-701, China).

\subsection{Blood Assay}

Blood samples of these four groups females were collected pre- and post- 4 weeks aerobics for detecting the plasma levels of TG, TC, HDL-C, LDL-C, Apo-A and Apo-B by Shanghai Adicon clinical laboratories Inc (Shanghai, China) by Beckman Coulter AU 680, and the ratios of LDL-C/HDL-C and Apo-B/Apo-A were calculated. The plasma levels of chemerin and Lp-PLA (R\&D systems Inc, USA) were detected by ELISA according to the protocol.

\subsection{Statistical Analysis}

Data were analyzed using SPSS 18.0 for windows. Mean values of body weight, BMI and body fat percentage were compared using analysis of covariate (ANCOVA), and the post-pre difference of other data were analyzed using one-way ANOVA.

\section{Results}

\subsection{Molecular Weight Distribution and Amino Acid Composition of CP}

As shown in Table 1, the most component of our CP is the part of 500-180 Da molecular weight, holding 65.72\% of total components, and the number and weight average molecular weights are 277 and 301 respectively. The part of 1000-500 Da molecular weight is in the second (17.65\%), whose number and weight average molecular weights are 277 and 301 respectively. According to the results of the proportion (83.37\%) of oligopeptides under 
$1000 \mathrm{Da}$ as well as the number and weight average molecular weight, a conclusion was drawn that the CP we

used are high quality products.

Table 1. Molecular weight distribution of $\mathbf{C P}$

\begin{tabular}{cccc}
\hline $\begin{array}{c}\text { Range of molecular weight } \\
\text { (Da) }\end{array}$ & $\begin{array}{c}\text { Peak area percentage (\%, } \\
\boldsymbol{\lambda} \text { 220 } \mathbf{n m})\end{array}$ & Number average molecular weight (Da) & Weight average molecular weight (Da) \\
\hline$>2000$ & 0.15 & 2265 & 2288 \\
$2000-1000$ & 2.98 & 1227 & 1265 \\
$1000-500$ & 17.65 & 645 & 669 \\
$500-180$ & 65.72 & 277 & 301 \\
$<180$ & 13.51 & $/$ & $/$
\end{tabular}

In addition, the total amino acid of our CP reach 62.47g glutamic acid and leucine, accounting for $15.79 \mathrm{~g}$ and per $100 \mathrm{~g}$ CP, among which the most two amino acids are $\quad 9.07 \mathrm{~g}$ in $100 \mathrm{~g}$ CP respectively (Table 2).

Table 2. Amino acid composition of $\mathrm{CP}$

\begin{tabular}{cccc}
\hline Amino acid & Mass (g/100g CP) & Amino acid & Mass $\mathbf{( g / 1 0 0 g}$ CP) \\
\hline Glutamic acid & 15.79 & Threonine & 2.25 \\
Leucine & 9.07 & Tyrosine & 1.98 \\
Alaline & 6.30 & Arginine & 1.95 \\
Proline & 3.85 & Glycine & 1.80 \\
Aspartic acid & 3.69 & Methionine & 1.72 \\
Serine & 3.50 & Histidine & 1.14 \\
Valine & 3.22 & Lysine & 0.99 \\
Isoleucine & 2.42 & Cystine & 0.55 \\
Phenylalanine & 2.26 & &
\end{tabular}

total amino acid

62.47

\subsection{Influence of $\mathrm{CP}$ in Combination with Exercise on Body Weight, BMI and Body fat Percentage}

The decreases of body weight, BMI and body fat percentage were no significant difference between $\mathrm{CP}+\mathrm{E} 1$ group and E1 group. More obvious reduce of body fat percentage (from $29.44 \% \pm 2.65 \%$ to $27.93 \% \pm 2.56 \%$ vs from $27.95 \% \pm 3.38 \%$ to $27.47 \% \pm 3.88 \%, \mathrm{p}<0.05$ ) and no difference of decreased body weight and BMI were shown in $\mathrm{CP}+\mathrm{E} 2$ group compare to $\mathrm{E} 2$ group. In addition, the above three indicators declined more remarkably in $\mathrm{CP}+\mathrm{E} 2$ group than that of $\mathrm{CP}+\mathrm{E} 1$ group (Table 3 ).

Table 3. Effect of CP in combination with exercise on body weight, BMI and body fat percentage of overweight and slight obese females

\begin{tabular}{|c|c|c|c|c|c|}
\hline & & E1 (n=6) & CP+E1 (n=7) & E2 (n=6) & $\mathrm{CP}+\mathrm{E} 2(\mathrm{n}=7)$ \\
\hline \multirow{4}{*}{ Body weight (kg) } & pre & $62.82 \pm 4.58$ & $68.27 \pm 4.89$ & $61.82 \pm 3.57$ & $66.20 \pm 4.80$ \\
\hline & post & $61.27 \pm 4.27$ & $67.54 \pm 4.69$ & $61.07 \pm 4.03$ & $63.94 \pm 4.35$ \\
\hline & $\Delta$ post-pre & $-1.55 \pm 1.20$ & $-0.73 \pm 0.66$ & $-0.75 \pm 1.08$ & $-2.26 \pm 1.16^{\#}$ \\
\hline & pre & $23.35 \pm 2.19$ & $24.60 \pm 1.45$ & $22.57 \pm 1.50$ & $24.90 \pm 1.17$ \\
\hline \multirow{2}{*}{ BMI $\left(\mathrm{kg} / \mathrm{m}^{2}\right)$} & post & $22.78 \pm 2.24$ & $24.31 \pm 1.39$ & $22.28 \pm 1.45$ & $24.06 \pm 1.10$ \\
\hline & $\Delta$ post-pre & $-0.57 \pm 0.41$ & $-0.29 \pm 0.23$ & $-0.28 \pm 0.51$ & $-0.84 \pm 0.44^{\#}$ \\
\hline \multirow{2}{*}{$\begin{array}{c}\text { Body fat percentage } \\
(\%)\end{array}$} & post & $28.37 \pm 1.99$ & $28.93 \pm 2.32$ & $27.47 \pm 3.88$ & $27.93 \pm 2.56$ \\
\hline & $\Delta$ post-pre & $-1.33 \pm 1.30$ & $-0.64 \pm 0.67$ & $-0.48 \pm 0.74$ & $-1.51 \pm 0.70^{* \#}$ \\
\hline
\end{tabular}

The diet of these females kept unchanged during the 4 weeks. E1: exercise 1 group; CP+E1: corn peptides+ exercise 1 group; E2: exercise 2 group;

$\mathrm{CP}+\mathrm{E} 2$ : corn peptides+ exercise 2 group.

${ }^{*} \mathrm{p}<0.05$ vs corresponding exercise group, ${ }^{*} \mathrm{p}<0.05$ vs $\mathrm{CP}+\mathrm{E} 1$ group.

\subsection{Influence of $\mathrm{CP}$ in Combination with Exercise on Plasma Lipid and Plasma Levels of Chemerin and Lp-PLA}

As shown in Table 4, no difference were found among the four groups in plasma levels of TG, TC, LDL-C, HDL-C, Apo-A and Apo-B as well as LDL-C/HDL-C and Apo-B/Apo-A. The above indicators of most of the overweight and slight obese subjects were normal (normal plasma levels of TG, TC, LDL-C, HDL-C as well as Apo$\mathrm{A}$ and Apo-B in human were less than $1.7 \mathrm{mmol} / \mathrm{L}, 5.2$ $\mathrm{mmol} / \mathrm{L}$ and $3.37 \mathrm{mmol} / \mathrm{L}$ and more than $1.04 \mathrm{mmol} / \mathrm{L}$ as well as 1.03-2.03 g/L and 0.42-1.26 g/L, respectively). In addition, the post-pre difference of the plasma levels of chemerin and Lp-PLA 2 kept unchanged among the four groups.

\subsection{Influence of $\mathrm{CP}$ in Combination with Exercise on the above Blood Indicators of Some Individuals}

Although no difference of the above indicators among groups was found, there were considerable variations among individuals. Only one of the two abnormal subjects were found to improve plasma indicator in $\mathrm{CP}+\mathrm{E} 1$ group while both of the two abnormal females returned to normal plasma indicators in $\mathrm{CP}+\mathrm{E} 2$ group (Table 5 ). What interesting was that the decrease of plasma TG, TC and LDL-C were accompanied with the downregulation of plasma chemerin in the three females with abnormal plasma lipid (No.7, 22 and 23) and unimproved plasma lipid concomitant with slight enhancement of plasma chemerin (No.8), which suggested that there might be a 
relation between the improved plasma lipid index and the were not shown in plasma levels of Lp-PLA . reduction of the plasma level of chemerin. Similary results

Table 4. Effect of CP in combination with exercise on plasma lipid and plasma levels of chemerin and Lp-PLA ${ }_{2}$ of overweight and slight obese females

\begin{tabular}{|c|c|c|c|c|c|}
\hline & & E1 & $\mathrm{CP}+\mathrm{E} 1$ & E2 & $\mathrm{CP}+\mathrm{E} 2$ \\
\hline \multirow{3}{*}{ TG (mmol/L) } & pre & $1.00 \pm 0.64$ & $1.05 \pm 0.56$ & $0.95 \pm 0.28$ & $0.90 \pm 0.67$ \\
\hline & post & $1.12 \pm 0.71$ & $1.12 \pm 0.29$ & $0.81 \pm 0.22$ & $0.82 \pm 0.28$ \\
\hline & $\Delta$ post-pre & $0.11 \pm 0.27$ & $0.08 \pm 0.44$ & $-0.15 \pm 0.32$ & $-0.07 \pm 0.61$ \\
\hline \multirow{3}{*}{ TC (mmol/L) } & pre & $4.10 \pm 0.91$ & $4.09 \pm 0.40$ & $4.14 \pm 1.28$ & $3.62 \pm 0.71$ \\
\hline & post & $3.88 \pm 1.10$ & $3.88 \pm 0.62$ & $3.99 \pm 0.97$ & $3.63 \pm 0.93$ \\
\hline & $\Delta$ post-pre & $-0.22 \pm 0.34$ & $-0.21 \pm 0.50$ & $-0.16 \pm 1.23$ & $0.01 \pm 0.74$ \\
\hline \multirow{3}{*}{ HDL-C (mmol/L) } & pre & $1.29 \pm 0.18$ & $1.31 \pm 0.13$ & $1.40 \pm 0.38$ & $1.25 \pm 0.30$ \\
\hline & post & $1.20 \pm 0.25$ & $1.18 \pm 0.24$ & $1.41 \pm 0.31$ & $1.35 \pm 0.42$ \\
\hline & $\Delta$ post-pre & $-0.10 \pm 0.14$ & $-0.11 \pm 0.16$ & $0.00 \pm 0.35$ & $0.10 \pm 0.30$ \\
\hline \multirow{3}{*}{ LDL-C (mmol/L) } & pre & $2.41 \pm 0.76$ & $2.39 \pm 0.33$ & $2.36 \pm 0.76$ & $2.06 \pm 0.48$ \\
\hline & post & $2.29 \pm 0.88$ & $2.31 \pm 0.46$ & $2.31 \pm 0.67$ & $2.03 \pm 0.52$ \\
\hline & $\Delta$ post-pre & $-0.12 \pm 0.24$ & $-0.08 \pm 0.32$ & $-0.06 \pm 0.77$ & $-0.04 \pm 0.44$ \\
\hline \multirow{3}{*}{ Apo-A (g/L) } & pre & $1.29 \pm 0.15$ & $1.34 \pm 0.94$ & $1.38 \pm 0.39$ & $1.25 \pm 0.26$ \\
\hline & post & $1.15 \pm 0.19$ & $1.22 \pm 0.20$ & $1.36 \pm 0.38$ & $1.35 \pm 0.43$ \\
\hline & $\Delta$ post-pre & $-0.14 \pm 0.11$ & $-0.12 \pm 0.17$ & $-0.02 \pm 0.42$ & $0.10 \pm 0.32$ \\
\hline \multirow{3}{*}{ Apo-B (g/L) } & pre & $0.66 \pm 0.25$ & $0.63 \pm 0.09$ & $0.61 \pm 0.21$ & $0.52 \pm 0.10$ \\
\hline & post & $0.60 \pm 0.28$ & $0.58 \pm 0.11$ & $0.58 \pm 0.15$ & $0.50 \pm 0.11$ \\
\hline & $\Delta$ post-pre & $-0.06 \pm 0.07$ & $-0.05 \pm 0.08$ & $-0.03 \pm 0.20$ & $-0.02 \pm 0.09$ \\
\hline \multirow{3}{*}{ LDL-C/HDL-C } & pre & $1.97 \pm 0.96$ & $1.85 \pm 0.32$ & $1.74 \pm 0.33$ & $1.69 \pm 0.36$ \\
\hline & post & $2.05 \pm 1.18$ & $2.00 \pm 0.58$ & $1.75 \pm 0.38$ & $1.56 \pm 0.44$ \\
\hline & $\Delta$ post-pre & $0.09 \pm 0.25$ & $0.15 \pm 0.42$ & $0.01 \pm 0.23$ & $-0.13 \pm 0.23$ \\
\hline \multirow{3}{*}{ Аро-В/Аро-А } & pre & $0.43 \pm 0.10$ & $0.47 \pm 0.09$ & $0.46 \pm 0.09$ & $0.42 \pm 0.10$ \\
\hline & post & $0.42 \pm 0.07$ & $0.49 \pm 0.10$ & $0.45 \pm 0.13$ & $0.39 \pm 0.14$ \\
\hline & $\Delta$ post-pre & $-0.01 \pm 0.05$ & $0.01 \pm 0.05$ & $-0.01 \pm 0.07$ & $-0.03 \pm 0.07$ \\
\hline \multirow{3}{*}{ Chemerin (ng/ml) } & pre & $48.25 \pm 9.56$ & $65.30 \pm 22.85$ & $29.05 \pm 17.44$ & $46.05 \pm 24.92$ \\
\hline & post & $44.05 \pm 20.90$ & $42.82 \pm 14.66$ & $31.06 \pm 13.25$ & $41.64 \pm 10.44$ \\
\hline & $\Delta$ post-pre & $-4.20 \pm 18.17$ & $-22.47 \pm 20.96$ & $2.01 \pm 26.33$ & $-14.22 \pm 20.38$ \\
\hline \multirow{3}{*}{ Lp-PLA 2 (ng/ml) } & pre & $100.64 \pm 38.95$ & $79.51 \pm 35.87$ & $81.97 \pm 31.39$ & $54.33 \pm 20.23$ \\
\hline & post & $86.79 \pm 42.05$ & $81.81 \pm 30.41$ & $80.30 \pm 40.17$ & $74.06 \pm 38.49$ \\
\hline & $\Delta$ post-pre & $-13.85 \pm 41.77$ & $2.29 \pm 33.92$ & $-1.68 \pm 32.73$ & $19.72 \pm 46.91$ \\
\hline
\end{tabular}

E1: exercise 1 group; $\mathrm{CP}+\mathrm{E} 1$ : corn peptieds+ exercise 1 group; E2: exercise 2 group; $\mathrm{CP}+\mathrm{E} 2$ : corn peptides+ exercise 2 group.

Table 5. Abnormal plasma lipid index and plasma levels of chemerin and LP-PLA $A_{2}$ in some females

\begin{tabular}{|c|c|c|c|c|c|}
\hline & & \multicolumn{2}{|c|}{$\mathrm{CP}+\mathrm{E} 1$} & \multicolumn{2}{|c|}{$\mathrm{CP}+\mathrm{E} 2$} \\
\hline & & No. 7 & \begin{tabular}{l|} 
No. 8 \\
\end{tabular} & No.22 & No.23 \\
\hline \multirow{2}{*}{ Body weight (kg) } & pre & 71.4 & 66.1 & 61.5 & 71.0 \\
\hline & post & 71.1 & 65.6 & 61.0 & 67.4 \\
\hline \multirow{2}{*}{ BMI $\left(\mathrm{kg} / \mathrm{m}^{2}\right)$} & pre & 24.7 & 25.8 & 24.8 & 25.8 \\
\hline & post & 24.6 & 25.6 & 24.3 & 24.5 \\
\hline \multirow{2}{*}{ Body fat percentage (\%) } & pre & 27.9 & 31.9 & 30.5 & 28.0 \\
\hline & post & 27.6 & 31.1 & 30.0 & 25.3 \\
\hline \multirow{2}{*}{ TG (mmol/L) } & pre & 2.25 & 2.28 & 2.39 & 1.39 \\
\hline & post & 1.50 & 2.40 & 1.03 & 1.02 \\
\hline \multirow{2}{*}{ TC (mmol/L) } & pre & 4.70 & 5.90 & 2.90 & 6.70 \\
\hline & post & 3.71 & 5.94 & 3.14 & 4.59 \\
\hline \multirow{2}{*}{ LDL-C (mmol/L) } & pre & 2.70 & 3.91 & 1.96 & 3.85 \\
\hline & post & 2.23 & 4.02 & 1.99 & 2.68 \\
\hline \multirow{2}{*}{ Chemerin (ng/ml) } & pre & 95.52 & 58.66 & 69.05 & 77.94 \\
\hline & post & 37.26 & 75.79 & 42.52 & 52.96 \\
\hline \multirow{2}{*}{ Lp-PLA 2 (ng/ml) } & pre & 100.22 & 122.20 & 128.42 & 97.30 \\
\hline & post & 142.50 & 139.34 & 121.62 & 88.56 \\
\hline
\end{tabular}

\section{Discussion}

Our previous work has demonstrated that CP only when combined with aerobic exercise decreased the body weight, body fat and improved the plasma lipid of high fat induced obese rats [6], while the present study indicated that only frequent exercise (5 times a week, $1 \mathrm{~h}$ per time) did exert fat reduction effect on overweight and slight obese females when combined with CP.

It has been demonstrated that leucine, a branched chain amino acid, decreased body weight and food intake [21] and play an important role in treatment of obesity and metabolic syndrome [22]. CP we used has a high amount 
of leucine $(9.07 \mathrm{~g} / 100 \mathrm{~g} \mathrm{CP})$. Whether the role of $\mathrm{CP}$ is through some oligopeptides, leucine or both need further investigation.

As shown in results, the plasma levels of TG, TC, LDLC, HDL-C as well as Apo-A and Apo-B in most of the overweight and slight obese subjects were normal, which may be contributed to the steadiness of the above indicators after treatment with CP combined with exercise. Although no differences of the above indicators among groups were found, there were considerable improvements of the above parameters in some abnormal individuals which restored to normal such as increased TC, TC and LDL-C.

A significant positive correlation was found between chemerin and BMI, TC, TC and LDL-C levels, and chemerin showed a significant negative correlation with the level of HDL-C $[7,8,9]$. Ress $C$ et al. demonstrated that bariatric surgery resulted in the decrease of circulating chemerin level, which may be related to the weight loss and improvements of lipid and glucose metabolism [16]. Except bariatric surgery, 6-month combined strength and endurance exercise led to a significant reduction in serum chemerin in overweight or obese, non-diabetic individuals [23]. The effect of 8-week lifestyle intervention (a homebased diet and exercise program) on the decrease of circulating chemerin was also demonstrated in young obese adults [24] and a 12-week exercise significantly decreased serum chemerin level in overweight and obese adults with type 2 diabetes [25]. In our study, no difference of plasma chemerin was found in CP combined with exercise groups compared with their respective exercise control groups, suggested that the fat reduction role of $\mathrm{CP}$ combined with exercise on overweight and slight obese females was not related to plasma chemerin. We speculated that the discrepancy between our results with others might be resulted from no change of blood lipid parameters which were within normal ranges, but it needs further study. It's worth noting that there were obvious decreases of chemerin in three females who were accompanied with the return of abnormal TG, TC and LDL-C, and no change of chemerin in a female whose upregualted TG, TC and LDL-C kept stable after treatment with CP in combination with exercise. The data make us speculated that the improvement of abnormal blood lipid parameters might be associated with decreased plasma chemerin, thus achieving blood lipid improvement of CP in combination with exercise on overweight and slight obese females, but it needs verification.

As a good biomarker of cardiovascular risk in obese adults, Lp-PLA 2 is strongly associated with stroke and many others cardiovascular events, which can be seen in obesity. Weight loss by diet control in obese women resulted in the significant reduction of the plasma levels of Lp-PLA $_{2}$ [20]. In our study, plasma Lp-PLA $A_{2}$ kept unchanged after $\mathrm{CP}$ combined with exercise treatment compared with exercise control, suggested that the fat loss resulted from CP combined with exercise was not related to plasma $\mathrm{Lp}-\mathrm{PLA}_{2}$ in overweight and slight obese females. Lp-PLA $A_{2}$ were negatively related to Apo-A and strongly positively associated with Apo-B and Apo$\mathrm{B} / \mathrm{Apo}-\mathrm{A}$ ratio $[26,27]$. So we speculated that unchanged plasma Lp-PLA $A_{2}$ levels might be attributed to no changes of Apo-A, Apo-B and Apo-B/Apo-A ratio in our study, it also needs further exploration.

\section{Conclusions}

In conclusion, only when frequent aerobic exercise was performed did CP in combination with aerobic exercise promote significantly the decrease of body fat percentage in overweight and slight obese females. The fat loss resulted from $\mathrm{CP}$ in combination with exercise is not related to the plasma levels of chemerin and Lp-PLA which might be attributed to no change of blood lipid parameters within normal ranges in overweight and slight obese females. Although no difference of chemerin between groups, changes of plasma chemerin in individual females with abnormal lipid parameters suggested that CP in combination with exercise might reverse the abnormal plasma levels of TG, TC and LDL-C by decreasing the plasma levels of chemerin.

\section{Acknowledgement}

This work was supported by Capacity Progress Project of Science and Technology Commission of Shanghai Municipality (NO.11290503000).

\section{List of Abbreviations}

TG: triglyceride; TC: total cholesterol; HDL-C: high density lipoprotein-cholesterol; LDL-C: low density lipoprotein-cholesterol; Apo-A: apolipoprotein a; Apo-B: apolipoprotein b; Lp-PLA 2 Lipoprotein-associated phospholipase $\mathrm{A}_{2}$; $\mathrm{BMI}$ : body mass index; $\mathrm{CP}$ : $\mathrm{CP}$; $\mathrm{E}$ : exercise.

\section{References}

[1] Xiuxia Li LH, Longjia Chen: In vitro antioxidant activity of protein hydrolysates prepared from corn gluten meals. Journal of the Science of Food and Agriculture 2008, 88(9):1660-1668.

[2] Yang Y, Tao G, Liu P, Liu J: Peptide with angiotensin Iconverting enzyme inhibitory activity from hydrolyzed corn gluten meal. J Agric Food Chem 2007, 55(19):7891-7895.

[3] Ma ZL, Zhang WJ, Yu GC, He H, Zhang Y: The primary structure identification of a corn peptide facilitating alcohol metabolism by HPLC-MS/MS. Peptides 2012, 37(1): 138-143.

[4] Melo-Silveira RF, Fidelis GP, Viana RL, Soeiro VC, Silva RA, Machado D, Costa LS, Ferreira CV, Oliveira Rocha HA: Antioxidant and antiproliferative activities of methanolic extract from a neglected agricultural product: corn cobs. Molecules 2014, 19(4): 5360-5378.

[5] Bong HY, Kim JY, Jeong HI, Moon MS, Kim J, Kwon O: Effects of corn gluten hydrolyzates, branched chain amino acids, and leucine on body weight reduction in obese rats induced by a high fat diet. Nutr Res Pract 2010, 4(2): 106-113.

[6] Shunli Wang XL, Ru Wang, Xiaohui Wang, Shujie Lou: Effect of corn peptide ingestion and exercise on protein metabolism in skeletal muscle of obese rats and its possible mechanism. Acta Nutrimenta Sinica [in chinese] 2013, 35(6): 540-544.

[7] Fulop P, Seres I, Lorincz H, Harangi M, Somodi S, Paragh G: Association of chemerin with oxidative stress, inflammation and classical adipokines in non-diabetic obese patients. J Cell Mol Med 2014.

[8] Lorincz H, Katko M, Harangi M, Somodi S, Gaal K, Fulop P, Paragh G, Seres I: Strong correlations between circulating chemerin levels and lipoprotein subfractions in nondiabetic obese and nonobese subjects. Clin Endocrinol (Oxf) 2013.

[9] Sledzinski T, Korczynska J, Hallmann A, Kaska L, ProczkoMarkuszewska M, Stefaniak T, Sledzinski M, Swierczynski J: The increase of serum chemerin concentration is mainly associated 
with the increase of body mass index in obese, non-diabetic subjects. J Endocrinol Invest 2013, 36(6): 428-434.

[10] Schultz S, Beck-Sickinger AG: Chemerin and vaspin: possible targets to treat obesity? ChemMedChem 2013, 8(4): 549-559.

[11] Wang D, Yuan GY, Wang XZ, Jia J, Di LL, Yang L, Chen X, Qian FF, Chen JJ: Plasma chemerin level in metabolic syndrome. Genet Mol Res 2013, 12(4): 5986-5991.

[12] Rourke JL, Dranse HJ, Sinal CJ: Towards an integrative approach to understanding the role of chemerin in human health and disease. Obes Rev 2013, 14(3): 245-262.

[13] Yang M, Yang G, Dong J, Liu Y, Zong H, Liu H, Boden G, Li L: Elevated plasma levels of chemerin in newly diagnosed type 2 diabetes mellitus with hypertension. J Investig Med 2010, 58(7): 883-886.

[14] Yu S, Zhang Y, Li MZ, Xu H, Wang Q, Song J, Lin P, Zhang L, Liu Q, Huang QX et al: Chemerin and apelin are positively correlated with inflammation in obese type 2 diabetic patients. Chin Med J (Engl) 2012, 125(19): 3440-3444.

[15] Herova M, Schmid M, Gemperle C, Loretz C, Hersberger M: Low dose aspirin is associated with plasma chemerin levels and may reduce adipose tissue inflammation. Atherosclerosis 2014, 235(2): 256-262.

[16] Ress C, Tschoner A, Engl J, Klaus A, Tilg H, Ebenbichler CF, Patsch JR, Kaser S: Effect of bariatric surgery on circulating chemerin levels. Eur J Clin Invest 2010, 40(3): 277-280.

[17] Kolodgie FD, Burke AP, Skorija KS, Ladich E, Kutys R, Makuria AT, Virmani R: Lipoprotein-associated phospholipase A2 protein expression in the natural progression of human coronary atherosclerosis. Arterioscler Thromb Vasc Biol 2006, 26(11): 2523-2529.

[18] Jiaxing Liu XZ, Yongwen Qin, et al: lipoprotein-associated phospholipase A2 as a predictor for severity of coronary atherosclerosis (in Chinese) Acad J Sec Mil Med Univ 2006, 27(4): 391-395.

[19] Koenig W, Twardella D, Brenner H, Rothenbacher D: Lipoprotein-associated phospholipase A2 predicts future cardiovascular events in patients with coronary heart disease independently of traditional risk factors, markers of inflammation, renal function, and hemodynamic stress. Arterioscler Thromb Vasc Biol 2006, 26(7): 1586-1593.

[20] Tzotzas T, Filippatos TD, Triantos A, Bruckert E, Tselepis AD, Kiortsis DN: Effects of a low-calorie diet associated with weight loss on lipoprotein-associated phospholipase A2 (Lp-PLA2) activity in healthy obese women. Nutr Metab Cardiovasc Dis 2008, 18(7): 477-482.

[21] Cota D, Proulx K, Smith KA, Kozma SC, Thomas G, Woods SC Seeley RJ: Hypothalamic mTOR signaling regulates food intake. Science 2006, 312(5775): 927-930.

[22] Layman DK, Walker DA: Potential importance of leucine in treatment of obesity and the metabolic syndrome. J Nutr 2006, 136(1 Suppl): 319S-323S.

[23] Stefanov T, Bluher M, Vekova A, Bonova I, Tzvetkov S, Kurktschiev D, Temelkova-Kurktschiev T: Circulating chemerin decreases in response to a combined strength and endurance training. Endocrine 2014, 45(3): 382-391.

[24] Lee MK, Chu SH, Lee DC, An KY, Park JH, Kim DI, Kim J, Hong S, Im JA, Lee JW et al: The association between chemerin and homeostasis assessment of insulin resistance at baseline and after weight reduction via lifestyle modifications in young obese adults. Clin Chim Acta 2013, 421: 109-115.

[25] Kim SH, Lee SH, Ahn KY, Lee DH, Suh YJ, Cho SG, Choi YJ, Lee SY, Hong SB, Kim YS et al: Effect of lifestyle modification on serum chemerin concentration and its association with insulin sensitivity in overweight and obese adults with type 2 diabetes. Clin Endocrinol (Oxf) 2014, 80(6): 825-833.

[26] da Silva IT, Timm Ade S, Damasceno NR: Influence of obesity and cardiometabolic makers on lipoprotein-associated phospholipase A2 (Lp-PLA2) activity in adolescents: the healthy young cross-sectional study. Lipids Health Dis 2013, 12: 19.

[27] Hirschler V, Merono T, Maccallini G, Gomez Rosso L, Aranda C, Brites F: Association of lipoprotein-associated phospholipase A(2) activity with components of the metabolic syndrome in apparently healthy boys. Cardiovasc Hematol Agents Med Chem 2011, 9(2): 78-83. 